be the process started bs the raying, it is certainly a persistent one, and I have several times been struck by the improvement which has been maintained in patients who for holiday purposes or the like have been several weeks away from treatment. The softening of the skin referred to above is often the first indication of absorption, and may be the first good sign noticed. This was markedly so in a case of mine-that of a middle-aged married lady whose face had been for long hidebound in an expressionless mask of lupus non-exedens. This patient told me, long before any visible sign of improvement was present, that the skin felt more flexible round the mouth Later on, as improvement became manifest, it was interesting to observe a gradual return of the normal lines of the features with increasing command of expression. When I last saw this case, early in the present year, a complete cure seemed to be not far off. The nose, lips, and cheeks were free from disease, and mere remnants of the trouble were left in broken streaks on the forehead and over the angles of the jaws. In this case, be it remarked, the reaction had at no time been excessive, and the removal of the disease had been brought about without any ulceration. The softness and healthy appearance of the skin marking the site of former disease is quite remarkable, and there is no treatment which could better have given such complete absence of scarring.

Cases of lupus of the face and neck are often seen where, as the result of disease and of frequent scraping operations, large hypertrophic scars are left which are almost as unsightly as the remaining disease. Such cases are eminently suitable for this treatment, whereby not only the active disease is overcome but the accompanying scars are softened and much improved in appearance.

The great drawback to the application of this method for lupus is, of course, the time and patience required on the patient's part. The treatment is not to be recommended in all cases, even of extensive disease.

I would summarize the cases of lupus suitable for $x$-ray treatment as being: ( 1 ) Cases of disease in prominent parts, such as the face, where the mischief is too extensive for Finsen's method ; (2) some ulcerated cases ; (3) cases associated with unsightly scars; (4) some cases of disease of mucous membrane.

\section{Rodent Ulcer.}

If it were only for the results achieved in dealing with this distressing disease, the $x$-ray treatment deserves special recognition. Instances of its successtul employment in this field are multiplying on all hands, and my own experience has been equally gratifying. One may roughly divide rodent uleers into two broad groups : clinically - the ulcerated and the non-ulcerated. I believe the former class responds more readily to the treatment, although I have seen excellent results with the latter, which I shall describe later. I shall describe first the course of events in a typical ulcerated rodent as I have almost uniformly observed them in cases where the malady has not covered any very large area or extended deeply to underlying structures.

After two or three weeks treatment one can invariably detect some softening in the hard collar-like crater of the ulcer, and there will probably be slight erythema of the skin around coinciding with the mask aperture. Coincident with these changes one can observe the surface of the ulcer alter its appearance. The characteristic waxy pale-red colour gives place to a deeper tint, and soon becomes dark red. At the same time the smoothness disappears, and one can detect small granulations springing up from the surface. These granulations multiply apace, and after a time raise the base of the ulcer flush with the surrounding skin. In one case, that of an old but vigorous man of over 70 , who had a rodent ulcer on the nose the size of a shilling piece, these granulations proliferated with extraordinary rapidity, and soon formed a table-land raised one-sixteenth of an inch above the surface, requiring restraint with copper sulphate. While the granulations are forming the border undergoes still further softening and thinning, and finally appears no thicker than normal skin, while a thin blue epithelial lip is seen to be spreading from it over the surface of the ulcer. We thus come to deal with what is apparentiy a simple healthy ulcer from which all the characteristics of malignancy have disappeared, and this ulcer gradually heals in the ordinary way. The resulting scar is marvellously smooth and supple. may occasionally happen that during the healing process the reaction becomes too strong. In two recent cases of this kind I have seen the newly-formed tissue break down again with distressing rapidity. The treatment was in both instances immediately suspended, and in one of them, when last seen, there was very evident healing again going on, while there was no suggestion of rodent ulcer to the naked eye. The other case was particularly interesting. There had been for some time a thick scab on the deepest part of the ulcer (which involved the upper lip and nasal orifice), and this I had not disturbed, though now I wish I had done so. It was under this scab that the breaking-down process first began, and on removing it I found a cousiderable quantity of pent-up discharge. This must have been rich in putrefying organisms, and could not but have proved deleterious to the granulation tissue. I am not without hope that this case, which up to the time of the relapse had healed in fully two-thirds of its extent, will in due time recover lost ground.

I have seen only one case-a very long-standing and extensive ulcer-which showed no apparent response whatever to the treatment. The non-ulcerated class of rodent ulcers are by no means insignificant in numbers, and not infrequently remain unrecognized until they have made considerable headway. They are characterized by the pale worm-like ridges, hard to the touch, which mark the advancing border of the disease, which proceeds in slowly.extending waves-their track shown by pale scar tissue more or less distinct.

Cases of this kind which I have observed have shown the usual reaction after a time. The ridges have first shown softening, sometimes being slowly absorbed without ulceration. Generally, however, they have broken down. The ulceration then marks out peculiarly well the sinuous lines which form the site of the thickened epithelium. Elsewhere the scar tissue becomes softer and redder, and finally the patches and streaks of ulceration heal in the ordinary

It still remains to be seen if the "cures" of rodent ulcer by this method will stand the test of time. The treatment is of too recent introduction for anything definitely to be said on this point. I can only remark that two cases of mine, which were discharged from treatment four and six months ago respectively, are still free from visible recurrence. It would probably be a wise precaution in all cases to continue the exposures for a time after apparent cure.

\section{THE CURATIVE EFFECT OF THE $X$ RAYS ON CALLOUS SINUSES OF THE ABDOMINAL WALL.}

BY D. BERRY HART, M.D., Gynaecologist to the Royal Infirmary, Edinburgh, etc.

Persistent sinuses of the abdominal wall where no foreign body is present are a great annoyance both to operator and patient. In two cases I have found these heal with marvellous rapidity after a few exposures to the $x$ rays, and thus think the experience worth recording for further trial. The fact of this action was stumbled on by accident as follows:

A patient, Mrs. S., was sent me from Singapore by Dr. Leask with a persistent sinus in the abdominal wall, after an incision in the right iliac fossa to evacuate bloody fluid of unknown origin. This sinus was 5 to 6 in. long, and ran from near the anterior iliac spine downwards and inwards to the uterus. It had existed for several months. In order, if possible, to see how it ran, I asked Dr. Dawson Turner, of the Edinburgh Infirmary Electrical Department, to take an $x$-ray photograph (a probe being placed in the sinus and one in the uterus). This did not help one much topographically as to the sinus, but I was struck with the almost magical cicatrization of the sinus after plugging with iodoform gauze for several weeks had failed.

In a second case of more marked sinus persisting for some months in a case of operations for extraperitoneal gestation

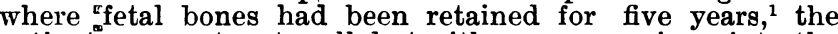
patient was sent out well but with a narrow sinus into the shrunk sac. This did not heal as I had hoped and the patient was again sent into Ward XXXVI with a sinus admitting the 
index finger. In my absence Dr. Milne Murray ordered the application of the $x$ rays and a cure was effected after three exposures. I have since learned indirectly that this sinus has reopened a little. It is possible there may be some bone present.

It seems to me advisable, therefore, to record these two cases so that a further trial may be given to a form of treatment now available in most large hospitals.

REFERENCE

1 Journal of Obstetrics and Gynaecology of the British Empire, No. 2, 1902.

\section{CASE OF SARCOMA OF THE FACE.}

Bx REGINALD J. GLADSTONE, M.D., F.R.C.S., Senior Demonstrator of Anatomy, Midjlesex Hospital.

IIN the following case a sarcoma of the face had extended from the antrum through the orbital cavity and sphenoidal fissure, and had exerted pressure on the temporo-sphenoidal lobe of the brain.]

It is rarely possible at a necropsy to obtain a satisfactory knowledge of the extent and character of malignant tumours of the face. The case which I now describe occurred in a female subject, aged 67, received at the Anatomical Department of the Middlesex Hospital. On inquiry I obtained the following particulars of her history :

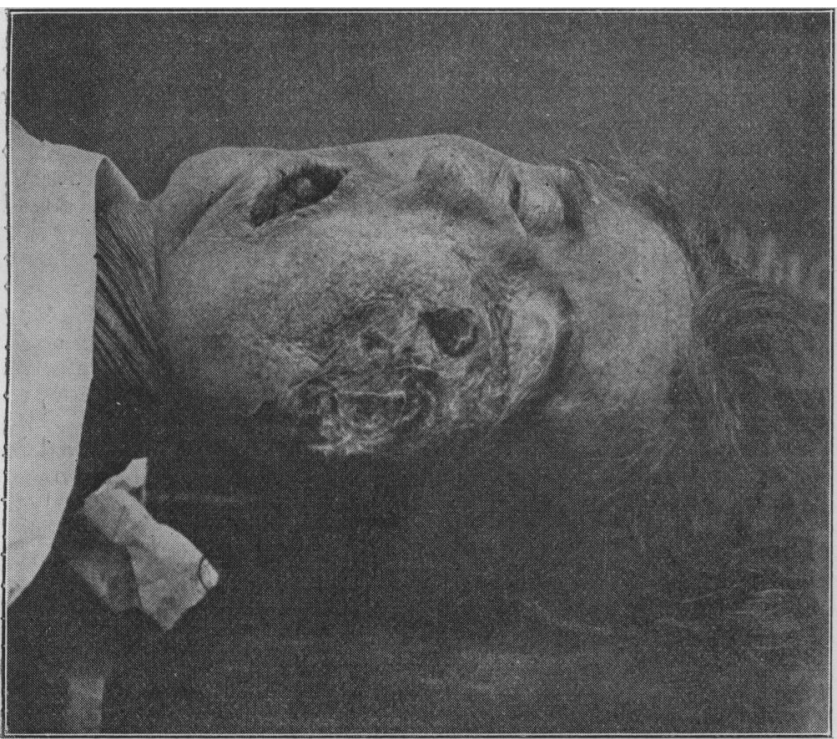

Fig. r.-Case of sarcema of the face. Surface view.

Y. Z., aged 67, a widow, employed as a needlewoman, occasionally intemperate, had suffered previously from bronchilis, ophthalmia, and aortic disease. She had been ailing for four months previous to admission with pain in the left side of the face, and some loss of flesh, and had also noticed a swelling in the same region for about three months. On examination there was found a painful swelling, with redness, on the left side of the face below the orbit. In side the moutb there was another swelling, which was commencing to fungate. Subsezjently the growth grew with great rapidity, ulcerating through the skiv, and nearly fllling the mouth. The left side of the face increased to an enormous size, and she finally died on August roth, roor of exhaustion.

From the above account, which was kindly given me by the resident medical officer of the infirmary in which the woman died, it appears that the symptoms were chiefly local, and that there were no obvious signs of pressure on the brain. The external appearance of the growth is well seen in the accompanying photograph (Fig. 1), which was taken after death. It will be noticed that the whole of the infraorbital part of the face on the left side, more especially in the region of the malar bone, is enormously enlarged, and that the growth has ulcerated through the skin in three separate places. On examining the eye the lower lid was found to be infiltrated with the growth and the eyeball pushed upwards and inwards.

The angle of the mouth was drawn downwards and outwards, and on examining the interior of the mouth it was found that a fungating mass of the growth had extended through the palate and had filled the whole of the upper part of the buccal cavity. On making a 'sagittal section through the frozen head, and removing the left side of the vault of the skull and the left cerebral hemisphere (Fig. 2), it was seen that the growth had extended backwards through the sphenoidal fissure into the middle cerebral fossa, forming a tumour there about the size of a walnut, which was beginning to fungate, and had pressed upon the temporal pole of the left cerebral hemisphere, indenting it, but not growing into it or causing any apparent injury to the brain substance.

On removing the roof of the orbital cavity the tumour was found to occupy the outer and posterior part of the fossa, having apparently reached it by growing upwards from the antrum through the orbital plate of the maxilla. The eyeball was displaced upwards and inwards, and the tumour was found to have grown backwards through the sphenoidal fissure below the lachrymal, frontal, and fourth cranial nerves, and the origins of the levator palpebrae and superior rectus muscles.

On examining the part exposed by the antero-posterior section of the head (Fig. 2), the growth was seen to have involved the greater part of the left nasal fossa, and also the sphenoidal air sinuses. The lateral mass of the ethmoid and the inferior turbinate bone were seen to be entirely replaced by the tumour, which had grown inwards from the maxillary sinus, and had pushed the nasal septum over to the right. That part of the tumour which had extended into the upper part of the mouth was also exposed by this section, and is well seen in the accompanying photograph.

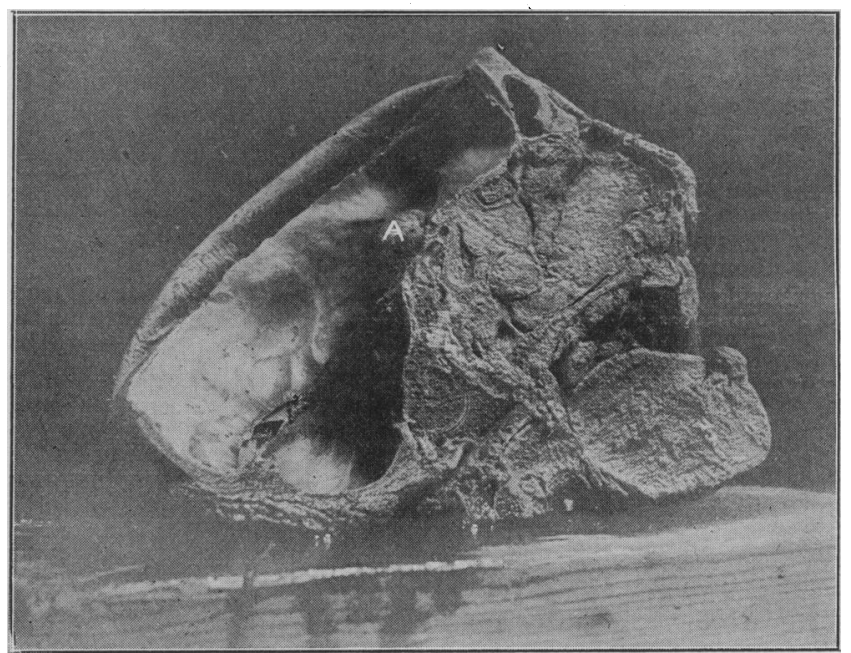

Fig. 2.-Sagittal ser.tion of head. A. Part of tumour which had grewn baskwards through the sphenoidal fissure.

A horizontal section through the most prominent part of the swelling showed that the tumour had broken down in the region of the antrum, the space being occupied by a loose blood clot. The tumour had the appearance of having originated at this spot, and of having spread from it to the regions mentioned above, and also into the spheno-maxillary and temporo-zygomatic regions.

The growth was very vascular, and composed chiefly of spindle and ovoid cells with large oval nuclei. The cells were packed close together, with little intercellular substance, and were contained in spaces the walls of which appear to have been formed by the connective tissue of the part which the tumour was invading.

CANCER IN ONTaRIo.-Cancer statistics in Ontario show an increase of 100 per cent. in the mortality between the years 189I and 1900 . The numbers of deatbs for the past ten years, beginning with 1891 were: 579, 676, 678, 621, 620, 731, 927, 975, I,04 I, I, 055.

Presentation.-At a jecent meeting of the Municipal Technical Institute Ambulance Class of Belfast, Dr. J. D. Williamson was presented with an address and a smoker's silver set by its members in appreciation of his valuable services as honorary lecturer. 